\title{
Facile fabrication of monodisperse fluorescent polystyrene composite microspheres
}

\author{
Zhenxiong $\mathrm{Ma}^{1,2, a}$, Ye Chen ${ }^{1,2}$, Qitao Zhang ${ }^{2}$, Teruhisa Ohno ${ }^{1, b_{*}}$, and Ming \\ Zhang ${ }^{2, c_{*}}$.
}

1Department of Applied Chemistry, Faculty of Engineering, Kyushu Institute of Technology, 1-1

Sensui, Tobata, Kitakyushu, Fukuoka 804-8550, Japan

\author{
2School of Chemistry and Chemical Engineering, Yangzhou University, Yangzhou 225002, \\ P.R.China \\ azxmayz@yahoo.com.cn, ${ }^{\mathrm{b}}$ tohno@che.kyutech.ac.jp, ${ }^{\mathrm{C} I x y z h a n g m @ y z u . e d u . c n}$ \\ * Corresponding authors
}

\begin{abstract}
Keywords: Fluorescent microsphere; Europium coordination compound; Polystyrene composite microsphere.
\end{abstract}

\begin{abstract}
Fluorescent poly(St-co-Eu(MAA) ${ }_{3}$ Phen) microspheres have been facilely fabricated via copolymerization of styrene and $\mathrm{Eu}(\mathrm{MAA})_{3}$ Phen with using the mixture of water and methanol as the dispersing medium in presence of sodium p-styrenesulfonate (NaSS). Strong photoluminescent europium coordination compound was inherited in the resulting polystyrene composite The microstructures and morphologies of the obtained poly(St-co-Eu(MAA) ${ }_{3}$ Phen) were characterized by using FT-IR, SEM-EDS, ICP-MS and TEM. Their fluorescence properties were measured by the fluorescence spectrometer.
\end{abstract}

\section{Introduction}

Nowadays, luminescent materials have attracted increasing attention. It is supposed to be an effective way to obtain excellent luminescent properties by rare earth doping. Generally, majority of rare earth ions have abundant f-orbital configurations, so there are three types of electronic transition in rare earth complex: $\mathrm{f}-\mathrm{f}$ transition, $\mathrm{f}-\mathrm{d}$ transition, and ligand-metal $\mathrm{f}$ charge transfer transition. The photophysical properties of rare earth ions such as Eu(III),[1-4]possessing longlived excited states and line-like emission bands, makes these ions as the excellent candidates for the applications such as photoelectric devices, solid state laser, optical data storage, flat plane displays, high energy radiation detectors and so on[5-7]. Moreover, fluorescent microsphere is a typical example, which has been used as fluorescent probes and luminescent labels in chemical, biological systems, and medical diagnostics[8].

To fabricate fluorescent microspheres with rare earth-polymer complexes, there are mainly two chemical routes to embed the metal-containing coordination chromophores covalently to polymeric bodies so as to prevent phase separation and leach photoactive components[9-11]: one is to apply a functional polymer to react with a metal salt and the other is to directly co-polymerize a metal-containing precursor[12, 13]. In ever reports, the resulting ionic aggregates in rare earth-polymer complexes tend to bring about critical emission quenching by the former way due to the fact that polymer chain could be tangled even at low rare earth content, while the latter method actually offers a well-defined structure around the metal center to lead to explicit optical-physics properties in turn, although the rare earth complexes are not just on the surface. However, there are still some problems in using such rare earth coordination compounds directly, for example-such complexes are insoluble in usual organic solution during polymerization.

Here, we present a facile way to fabricate stable fluorescent microsphere via the copolymerization of styrene with Eu(MAA) ${ }_{3}$ Phen by using sodium p-styrenesulfonate (NaSS) and polyvinylpyrrolidone(PVP) K90 as stabilizers, N,N'-dimethylformamide(DMF) as co-solvent, which leads to the presence of fluorescent europium complexes on the surface of microspheres, and 
generating new composite fluorescent markers with enhanced stability.

\section{Experimental}

\section{Materials.}

Styrene (monomer, St) was purchased from Kanto Chemical Co., Inc., Japan. Eu(MAA) ${ }_{3}$ Phen (synthesized in our laboratory).Sodium p-styrenesulfonate (NaSS), potassium persulfate (KPS) were purchased from Wako Pure Chemicals Co., Japan. Polyvinylpyrrolidone(PVP) K90 was purchased from Tokyo chemical industry Co. Ltd., Japan.

\section{Synthesis of poly(St-co- Eu(MAA) $)_{3}$ Phen) microspheres.}

Poly(poly(St-co-Eu(MAA) ${ }_{3}$ Phen) microspheres were synthesized using the co-polymerization technique in a four-neck mercury-sealed round-bottom flask fitted with a reflux condenser, Teflon stirrer powered by a high-torque mechanical stirrer and nitrogen and reagent inlets. The temperature was maintained by placing the reaction vessel in a controlled-temperature oil bath. The reaction vessel was charged with double-distilled water and methanol. The stirring rate was kept at $300 \mathrm{rpm}$. A nitrogen blanket was maintained throughout the reaction. The solution was deoxygenated by bubbling with nitrogen for 2 hrs. After a thorough deoxygenation, NaSS dissolved in distilled water, styrene, Eu(MAA) ${ }_{3}$ Phen, methanol, DMF and the solution of polyvinylpyrrolidone K90 were injected and the temperature was increased to $70^{\circ} \mathrm{C}$. After equilibration for $30 \mathrm{~min}$, KPS dissolved in water was injected into the reaction mixture. The temperature and stirring rate were then maintained for $10 \mathrm{hrs}$ to complete reaction.

The un-grafted monomer and residuals were removed by ultracentrifugation of microspheres at $10,000 \mathrm{rpm}$ for $2 \mathrm{hrs}$ and washed by distilled water for repeating 3 times at least.

\section{Characterization.}

Fluorescence experiments were performed with the help of F-4500 FL Spectrophotometer (Hitachi, Japan). The infrared spectrum was recorded with FT-IR 460 (JASCO, Japan) using a KBr pellet. The transmission electron micrographs of products were examined by Tecnai-12 (Philip Apparatus Co., Netherlands.). The determination of the amount of europium was performed with the help of Elan DRC-e coupling plasma mass spectrometer (PerkinElmer, U.S.A)

\section{Results and discussion}

Fig. 1 showed the stretching vibration of Phen at $1611 \mathrm{~cm}^{-1}$ and the bent vibrations at 848 and $752 \mathrm{~cm}^{-1}$ respectively.

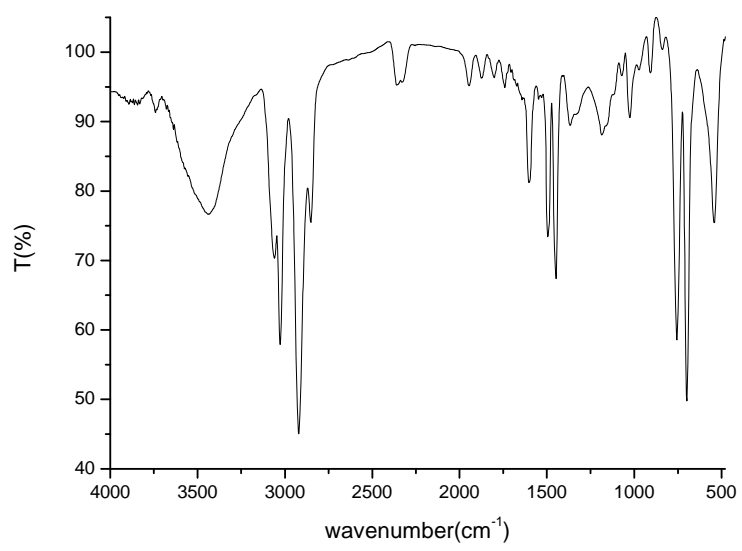

Fig. 1 FT-IR of poly(St-co- Eu(MAA) $)_{3}$ phen) microsPheres

The peak around $1029 \mathrm{~cm}^{-1}$ represents the symmetric stretching vibration of the $\mathrm{SO}_{3}$ group and the relatively broad peak around $1177 \mathrm{~cm}^{-1}$ represents the asymmetric vibration of the $\mathrm{SO}_{3}$ group, indicating that there is $\mathrm{SO}_{3}{ }^{-}$on the surface of poly(St-co-Eu(MAA) ${ }_{3}$ Phen) microspheres. The absorption peak at $1727 \mathrm{~cm}^{-1}$ assigns to the stretching vibrations of carbonyl group, and the bands at $1566 \mathrm{~cm}^{-1}$ vas $\left(\mathrm{COO}^{-}\right)$and $1431 \mathrm{~cm}^{-1}$ vs $\left(\mathrm{COO}^{-}\right)$could confirm that Eu(MAA) $)_{3}$ Phen was 
successfully copolymerized with styrene onto the polymer microspheres, too.

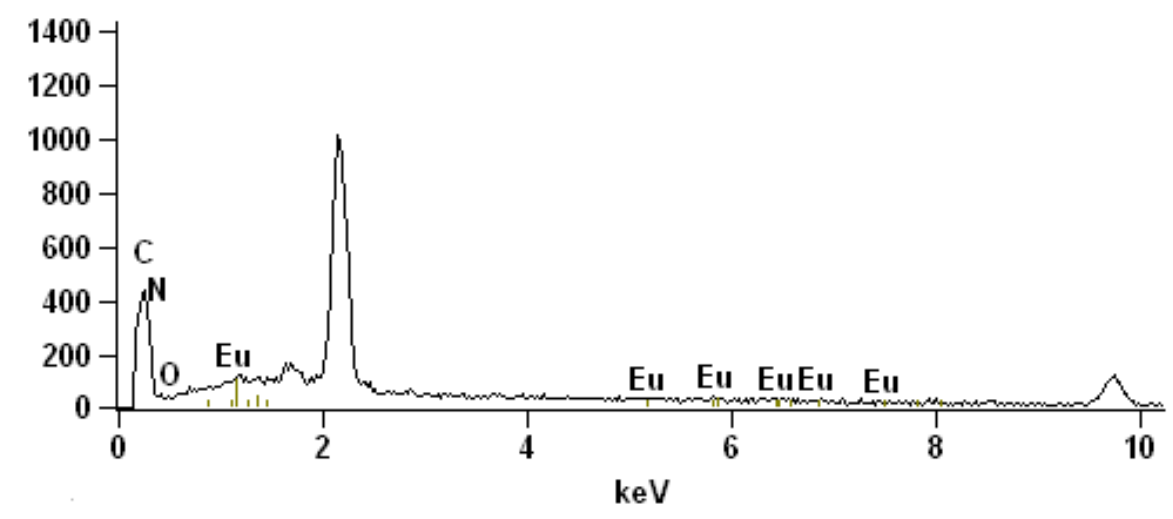

Fig. 2 SEM-EDS spectrum of poly (St-co-Eu(MAA) ${ }_{3}$ Phen) microspheres

To get a comprehensive understanding of europium content in the microspheres, we have used the same dried poly(St-co-Eu(MAA) $)_{3}$ Phen) powers to check the results of SEM-EDS and ICP-MS. From Fig.2, there is only a little amount of europium element in poly(St-co-Eu(MAA) $)_{3}$ Phen) microspheres. The result of SEM-EDS $(0.21 \%)$ is less than that of ICP-MS( $0.39 \%)$, maybe it is due to not only the lack of representative of analytical area in SEM-EDS but also that there are europium complexes both in core and on the surface of the microspheres. hence the result of ICP-MS presents the whole amount of europium.

In this paper, NaSS does polarity and stability of the growing particles during the polymerization. $\mathrm{NaSS}$ forms an ionic charge on the particle surface which can repel the particles and prevent agglomeration. The NaSS oligomers generated in the aqueous phase in the first place will capture styrene monomer droplets until the radicals exceed their solubility and precipitate. The aggregated radicals absorb monomers and then polymerize. As there are no micelles above all to capture such oligoradicals, hence they will keep on growing.

As a result, the particle size decreased when NaSS concentration increased. (Fig.3) (from 974nm to $1148 \mathrm{~nm}$ ) The particles prepared in presence of NaSS are much smaller than those prepared without NaSS, because an oligomer bearing more charge groups should be more rigid to nucleate or to adsorb on the likely charged particle surfaces.

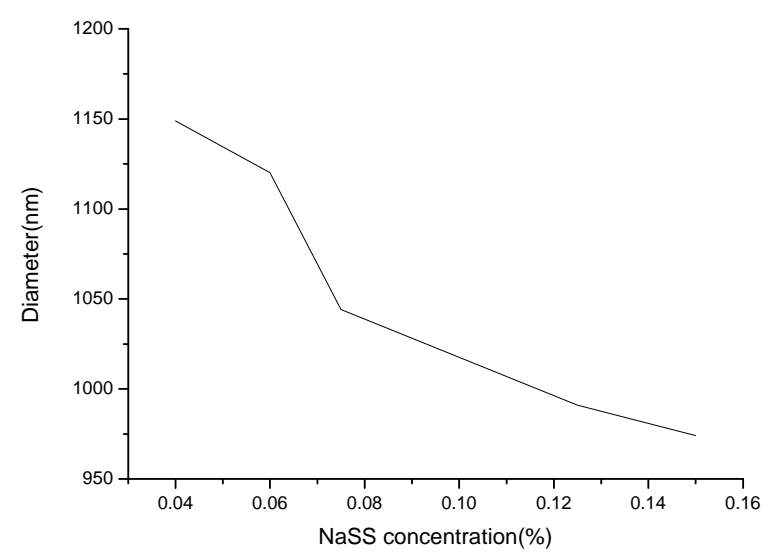

Fig. 3. Variation of particle size vs. NaSS concentration

PVP K90 is essential to this polymerization to obtain monodisperse microsphere, moreover there is a synergistic effect between KVP and NaSS to stabilize the formed particles. When PVP concentration increased, the composite particles varied from polydisperse to monodisperse(Fig.4), here PVP formed a homologous colloidal to disperse the particles well and avoid the agglomeration of them. 

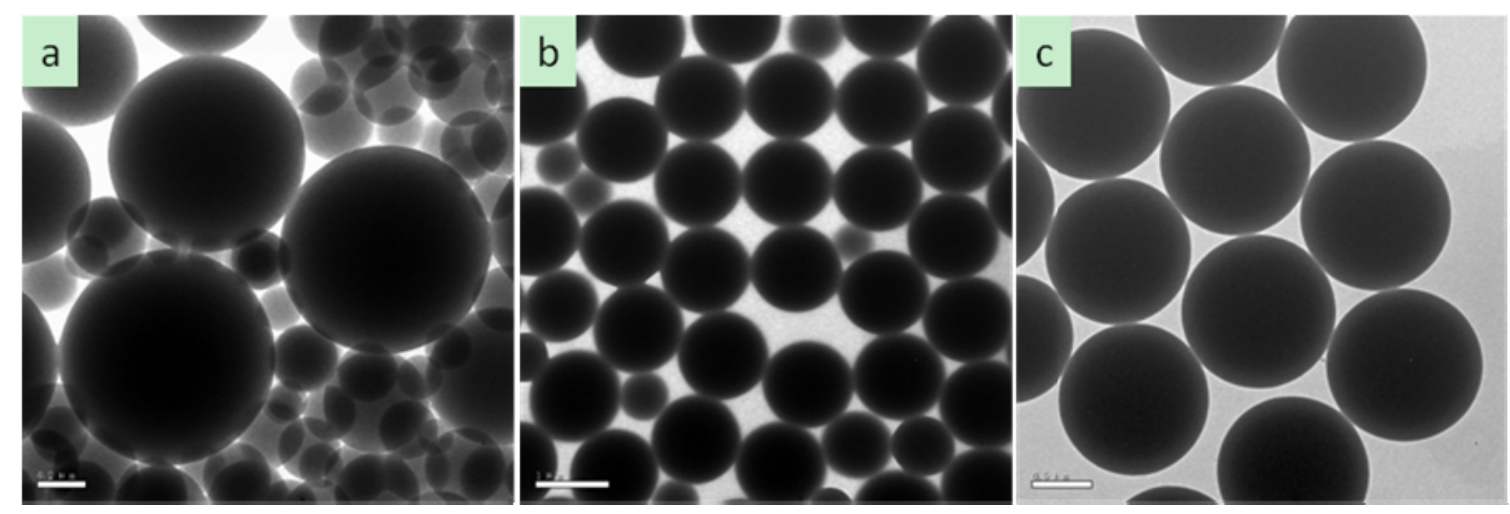

Fig. 4. TEM images of particle vs. PVP K90 concentration. (PVP concentration: a $0.01 \%$, b 0.03\%, c $0.05 \%)$

The fluorescent properties of poly(St-co-Eu(MAA) $)_{3}$ Phen) were measured on fluorescent spectrometer. Fig. 5 shows the excitation (left) and emission (right) spectrum of the microspheres. In the excitation spectrum of poly(St-co-Eu(MAA) $\left.)_{3} \mathrm{Phen}\right)$, a broadband at $292 \mathrm{~nm}$ is observed, assigned to the absorption from ligand, which indicated that there was energy transfer between the surrounding ligand to $\mathrm{Eu}^{3+}$, someway confirmed that $\mathrm{Eu}(\mathrm{MAA})_{3}$ Phen was successfully copolymerized onto the surface of composite microspheres..

Upon excitation at the maximum absorption $(292 \mathrm{~nm})$, characteristic luminescent bands of europium (III) exhibit at $569\left({ }^{5} \mathrm{D}_{0} \rightarrow{ }^{7} \mathrm{~F}_{0}\right), 585\left({ }^{5} \mathrm{D}_{0} \rightarrow{ }^{7} \mathrm{~F}_{1}\right), 619\left({ }^{5} \mathrm{D}_{0} \rightarrow{ }^{7} \mathrm{~F}_{2}\right)$, and $699 \mathrm{~nm}\left({ }^{5} \mathrm{D}_{0} \rightarrow{ }^{7} \mathrm{~F}_{4}\right)$ in the emission spectrum, which is in agreement with the previous reports.[14-16] Because of the shielding of the $4 \mathrm{f}$ orbital by an outer shell of the $5 \mathrm{~s}$ and $5 \mathrm{p}$ orbitals, the $\mathrm{f}-\mathrm{f}$ absorption bands of $\mathrm{Eu}^{3+}$ are very narrow. This transition is responsible for the red color emitted by the composite microspheres.

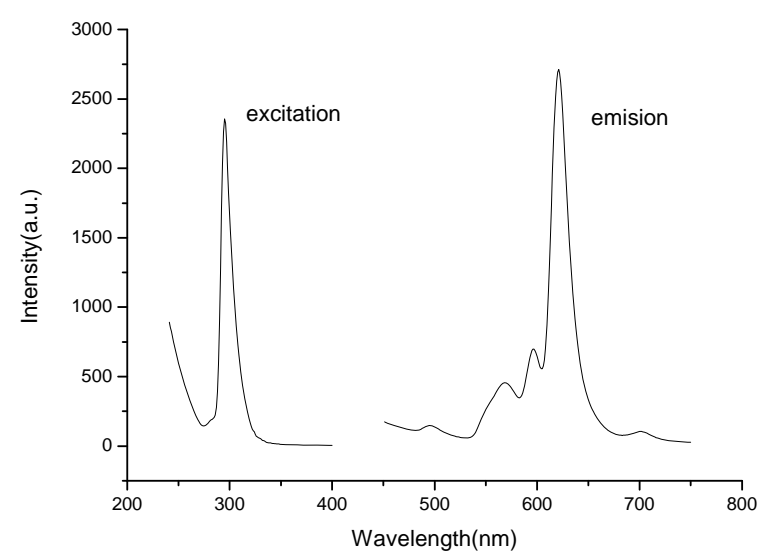

Fig. 5 Fluorescent spectra of poly(St-co-Eu(MAA) $)_{3}$ Phen) microspheres(solvent: DMSO)

Commonly, the ${ }^{5} \mathrm{D}_{0} \rightarrow{ }^{7} \mathrm{~F}_{1}$ transition as magnetic dipolar transition is insensitive to the surrounding environment while ${ }^{5} \mathrm{D}_{0} \rightarrow{ }^{7} \mathrm{~F}_{2}$ is electric dipolar transition, therefore, the relative intensity ratio of ${ }^{5} \mathrm{D}_{0} \rightarrow{ }^{7} \mathrm{~F}_{2}$ to ${ }^{5} \mathrm{D}_{0} \rightarrow{ }^{7} \mathrm{~F}_{1}$ could value that the local environment of the europium(III) ions is centrosymmetric or not.

Among them, the emission intensity from the ${ }^{5} \mathrm{D}_{0} \rightarrow{ }^{7} \mathrm{~F}_{2}$ transition at $619 \mathrm{~nm}$ was remarkably higher than those of the other three peaks. The reason why the composite microspheres emit strong fluorescence could be attributed to the excellent matching of energy levels between the ligands and the central ion $\mathrm{Eu}^{3+}$, the ligands absorb ultraviolet photons and are excited; by intersystem crossing, effective energy transfer between the ligands and the central ion $\mathrm{Eu}^{3+}$ occurs.

\section{Conclusions}

A novel luminescent microsphere was synthesized in ordinary condition. The microspheres with europium complex on their surface were proved to be excellent luminous materiasl. This is 
attributed to its high emission intensities, caused by the stable coordination structure originated from $\mathrm{Eu}(\mathrm{MAA})_{3}$ Phen on the surface of the poly(St-co-Eu(MAA) $)_{3}$ Phen) microsphere, in which the effective energy transfer from Phen ligand to $\mathrm{Eu}^{3+}$ ions occurred indeed, as evidenced by the results of fluorescence spectrum.

The preparation and characterization of poly(St-co-Eu(MAA) $)_{3}$ Phen) microspheres are presented. To our knowledge, this is the first publication, describing the preparation and application of luminescent composite polystyrene microspheres based on the copolymerization of styrene with $\mathrm{Eu}(\mathrm{MAA})_{3}$ Phen. Good fluorescent microspheres with high intensity can be prepared directly without any further modification. The current method may be extended to other rare earth complexes for the preparation of functional microspheres in similar medium.

\section{References}

[1] Q. M. Wang and B. Yan: J. Photochem. Photobiol. A 177 (2006), p. 1.

[2] Y. Kobayashi, J. Imai, D. Nagao, and M. Konno: Colloid Surf. A-Physicochem. Eng. Asp. 326 (2008), p. 109.

[3] P. Lenaerts, C. Gorller-Walrand, and K. Binnemans: J. Lumin. 117 (2006), p. 163.

[4] A. S. Ndao, A. Buzady, J. Erostyak, and I. Hornyak: J. Fluoresc 18 (2008), p. 649.

[5] R. Lange, K. Doblhofer, and W. Storck: Electrochim. Acta 33 (1988), p. 385.

[6] T. Pagnot, P. Audebert, and G. Tribillon: Chem. Phys. Lett. 322 (2000), p. 572.

[7] M. Andrews, R. H. Laye, L. P. Harding, and S. J. A. Pope: Polyhedron 27 (2008), p. 2365.

[8] P. Huhtinen, M. Kivela, O. Kuronen, V. Hagren, H. Takalo, H. Tenhu, T. Lovgren, and H. Harma: Anal. Chem. 77 (2005), p. 2643.

[9] K. Rao, H. G. Liu, and Y. I. Lee: Appl. Spectrosc. Rev. 45 (2010), p. 409.

[10] X. F. Qiao and B. Yan: Inorg. Chem. 48 (2009), p. 4714.

[11] N. V. Petrochenkova, B. V. Bukvetskii, A. G. Mirochnik, and V. E. Karasev: Russ. J. Coord. Chem. 28 (2002), p. 64.

[12] C.-S. Zhao, X.-L. Liu, M. Yang, J.-Y. Fang, J.-J. Zhang, and F.-Q. Liu: Dyes. Pigments. 82 (2009), p. 134.

[13] C. X. Du, L. Ma, Y. Xu, and W. L. Li: J. Appl. Polym. Sci. 66 (1997), p. 1405.

[14] V. Tsaryuk, V. Zolin, and J. Legendziewicz: J. Lumin. 102 (2003), p. 744.

[15] S. I. Klink, G. A. Hebbink, L. Grave, P. Alink, F. van Veggel, and M. H. V. Werts: J. Phys. Chem. A 106 (2002), p. 3681.

[16] O. L. Malta and F. Silva: Spectrochim. Acta. A 54 (1998), p. 1593. 